\title{
LIST OF FORTHCOMING PAPERS
}

for the special issues 'New Windows of the Universe'

J. A. Belmonte, A. R. Jones, P. L. Pallé, and T. Roca Cortés: Acoustic Oscillations in the K2 III Star Arcturus. (Received 30 January, 1990.)

M. Hernanz, J. Isem, R. Canal, and J. Labay: Precollapse Evolution of Accreting Co White Dwarfs. (Received 30 January, 1990.)

E. Bravo, J. Isern, R. Canal, and J. Labay: Nucleosynthesis in SN Ia. (Received 30 January, 1989.)

D. García, J. Labay, R. Canal, and J. Isern: Dynamical Instability in Accreting White Dwarfs. (Received 30 January, 1990.)

V. F. Polcaro, C. Rossi, L. Norci, and F. Giovannelli: The Unusual Star V439 CYG. (Received 30 January, 1990.)

S. Gilheany, B. Bates, M. G. Catney, and P. L. Dufton: Stellar Mass Loss in Late B-Type Supergiants. (Received 30 January, 1990.)

Roberto Viotti, Giovanni Battista Baratta, Anna Rosa Marenzi, and Marcello Ranieri: The Nature of $\eta$ Carinae. (Received 30 January, 1990.)

E. Mediavilla, J. Buitrago, and M. Portilla: Radiation Forces and the Formation of Planetary Systems. (Received 30 January, 1990.)

F. Giovanelli, C. Rossi, D. Castaldo, E. Covino, L. Errico, A. A. Vittone, G. S. Bisnovatyi-Kogan, V. G. Kurt, S. A. Lamzin, and E. K. Sheffer: Multifrequency Monitoring of the Pre-MainSequence Star RU Lupi. (Received 30 January, 1990.)

E. Paez, Roger C. Martinez, and O. Straniero: Deep CCD Photometry of Stars in the Globular Cluster M3. (Received 30 January, 1990.)

A. Herrero, R. P. Kudritzki, and J. M. Vilchez: NLTE Analysis of Massive OB stars in Open Clusters. (Received 30 January, 1990.)

R. J. García Lopez, R. Rebolo, J. E. Beckman, and C. D. McKeith: The HE I $D_{3} 5876 \AA$ Line as an Activity and Structural Indicator in F-Type Hyades Stars. (Received 30 January, 1990.)

F. Giovannelli, I. Gonzalez Martinez-Pais, S. Gaudenzi, R. Lombardi, C. Rossi, and R. U. Claudi: Multifrequency Behaviour of the Dwarf Nova SS Cygni. (Received 30 January, 1990.)

A. Herrero, R. H. Méndez, and A. Manchado: NLTE Analysis of High-Resolution Spectra of CSPN. (Received 30 January, 1990.)

V. Piirola, G. V. Coyne, and A. Reiz: Multicolour Photopolarimetry of Magnetic Cataclysmic Variables. (Received 30 January, 1990.)

F. Giovannelli, C. Bartolini, A. Guamieri, A. Piccioni, M. Burger, E. L. Van Dessel, V. G. Kurt, E. K. Sheffer, D. De Martino, R. Waters, I. Gonzales Martinez-Pais, Yu. N. Gnedin, V. M. Larionov, and N. I. Shakovskaya: Fourteen Years of Multifrequency Coordinated Observations of the X-Ray/Be System A $0535+26 / \mathrm{HDE} 245770$. (Received 30 January, 1990.)

P. A. Charles, M. J. Arévalo, J. Baruch, R. Biernicowicz, P. Callanan, J. Casares, V. Dhillon, A. Giménez, I. González, R. González, E. Harlaftis, B. Hassall, C. Hellier, P. Johnson, D. Jones, M. Kidger, C. Lázaro, K. Mason, K. Mukai, T. Naylor, V. Reglero, R. Rutten, J. L. Sedano, and J. Van Paradijs: Accretion Disc Evolution in Cataclysmic Variables (The 1988 International Time Project in La Palma). (Received 30 January, 1990.)

F. J. Barrón De Angoiti: Final Evolutionary Stages of 8-10 $M_{\odot}$ Stars. (Received 30 January, 1990.) 